\title{
REGULARIZATION AND APPROXIMATION OF LINEAR OPERATOR EQUATIONS IN REPRODUCING KERNEL SPACES ${ }^{1}$
}

\author{
BY M. Z. NASHED AND GRACE WAHBA
}

Communicated by Michael Golomb, May 13, 1974

1. Introduction. Let $X$ and $Y$ be real Hilbert spaces and let $A$ be a linear operator with domain $D(A) \subset X$ and range in $Y$. An element $u \in$ $D(A)$ is said to be a least-squares solution of the equation

$$
A x=y
$$

for a given $y \in Y$ if $\inf \{\|A x-y\|: x \in D(A)\}=\|A u-y\|$. A pseudosolution of (1) for a given $y \in Y$ is a least-squares solution of minimal norm. Equation (1) is well-posed relative to the spaces $X, Y$ if for each $y \in Y$, (1) has a unique pseudosolution which depends continuously on $y$; otherwise the equation is said to be ill-posed.

One objective of this research is to show, when $X$ and $Y$ are $L_{2}$ spaces of square-integrable functions, that the topology of reproducing kernel Hilbert spaces (RKHS) is an appropriate topology for the regularization of ill-posed linear operator equations, and to initiate a study of generalized inverses of linear operators acting between two RKHS. A second objective is to provide an approach to optimal approximations of linear operator equations in the context of RKHS, and to demonstrate the relation between the regularization operator of the equation $A f=g$ and the generalized inverse of $A$ in an appropriate RKHS. (For some background on regularization methods see [3], [5], [9] ; for generalized inverses see, for example, [4] .)

AMS (MOS) subject classifications (1970). Primary 47A50, 65J05, 46G22, 65J05, $41 \mathrm{~A} 25$; Secondary 65D99, 65F20, 45L10.

Key words and phrases. Ill-posed problems, linear operator equations, regularization, reproducing kernel spaces, generalized inverses, moment discretization, convergence rates.

${ }^{1}$ Research sponsored by the United States Army under Contract No. DA-31-124-ARO-D-462. 
2. Generalized inverses in RKHS. Let $H_{Q}$ denote the RKHS of realvalued functions on the bounded interval $S$ with reproducing kernel $Q\left(s, s^{\prime}\right)$. Denote the inner product and norm in $H_{Q}$ by $\langle\cdot, \cdot\rangle_{Q}$ and $\|\cdot\|_{Q}$ respectively. Then for $f \in H_{Q}, f(s)=\left\langle Q_{s^{\prime}} f\right\rangle_{Q}$ where $Q_{s}\left(s^{\prime}\right):=Q\left(s, s^{\prime}\right)=$ $\left\langle Q_{s}, Q_{s^{\prime}}\right\rangle$ for $s, s^{\prime} \in S$. (See [1] for properties of RKHS.) The kernel $Q\left(s, s^{\prime}\right)$ induces a selfadjoint Hilbert-Schmidt operator on $L_{2}[S]$, the space of square-integrable real-valued functions on $S$, by

$$
(Q f)(s)=\int_{S} Q\left(s, s^{\prime}\right) f\left(s^{\prime}\right) d s^{\prime}
$$

Furthermore, we have $H_{Q}=Q^{1 / 2}\left(L_{2}[S]\right)$ and

$$
\|f\|_{Q}=\inf \left\{\|p\| L_{2}[S]: p \in L_{2}[S], Q^{1 / 2} p=f\right\}
$$

(cf. [8], [10]). For $f \in H_{Q}$, let $Q^{-1 / 2} f$ denote the element $p$ of minimal $L_{2}[S]$-norm that satisfies $Q^{1 / 2} p=f$. We then have

$$
\left\langle f_{1}, f_{2}\right\rangle_{Q}=\left\langle Q^{-1 / 2} f_{1}, Q^{-1 / 2} f_{2}\right\rangle L_{2}[S]
$$

THEOREM 1. Let $A$ be a linear operator from $X=L_{2}[S]$ into $Y=$ $L_{2}[T]$, where $S, T$ are closed bounded intervals. Assume that $A$ has the following properties:

(i) $H_{Q} \subset D(A) \subset X$ (throughout " $\subset$ " denotes point-set inclusion only), where $H_{Q}$ is an RKHS with continuous kernel on $S \times S$;

(ii) $A\left[H_{Q}\right] \subset H_{R} \subset H_{\widetilde{R}} \subset Y$, where $H_{R}$ and $H_{\widetilde{R}}$ are $R K H S$ with continuous kernels on $T \times T$; and

(iii) the null space of $A$ in $H_{Q}$ is closed in $H_{Q}$.

Let $A_{X, Y}^{\dagger}\left(\right.$ resp. $\left.A_{Q, \widetilde{R}}^{\dagger}\right)$ denote the generalized inverse of $A$ when $A$ is considered as a map from $X$ into $Y$ (resp. from $H_{Q}$ into $H_{\widetilde{R}}$ ). Let $y \in D\left(A_{Q, \widetilde{R}}^{\dagger}\right)$. Then $\left.y \in D\left(Q^{1 / 2} A Q^{1 / 2}\right)_{(X, Y)}^{\dagger} \widetilde{R}^{-1 / 2}\right)$ and

$$
A_{(Q, \widetilde{R})}^{\dagger} y=Q^{1 / 2}\left(\widetilde{R}^{-1 / 2} A Q^{1 / 2}\right)_{X, Y}^{\dagger} \widetilde{R}^{-1 / 2} y .
$$

(The operators $Q$ and $\widetilde{R}$ are those induced by the RKHS $H_{Q}$ and $H_{\widetilde{R}}$ respectively.)

It should be noted that an operator $A$ may map $H_{Q}$ onto another RKHS, while failing to have a closed range in $L_{2}[T]$. This is, for example, the case if $A$ is a Hilbert-Schmidt integral operator (with nondegenerate kernel) on $L_{2}[S]$. 
3. Explicit representations of minimal-norm solutions of linear operator equations in RKHS. We assume that $H_{Q}$ is chosen so that

(3) the linear functionals $E_{t}$ defined by $E_{t} f=(A f)(t)$ are continuous in $H_{Q}$.

Then there exists $\eta_{t} \in H_{Q}$ for $t \in T$ such that $(A f)(t)=\left\langle\eta_{t}, f\right\rangle_{Q}$, where $\eta_{t}(s)=\left\langle\eta_{t}, Q_{s}\right\rangle_{Q}=A Q_{s}(t)$. Let $H_{R}$ be the RKHS with kernel $R\left(t, t^{\prime}\right):=\left\langle\eta_{t}, \eta_{t^{\prime}{ }^{\prime}}, t, t^{\prime} \in T\right.$. Let $V$ be the closure of the span of $\left\{\eta_{t}: t \in T\right\}$ in $H_{Q}$. It follows easily that the null space of $A$ in $H_{Q}$ is $V^{\perp}\left(\perp\right.$ in $\left.H_{Q}\right)$. Since $\left\langle\eta_{t}, \eta_{t^{\prime}}\right\rangle_{Q}=\left\langle R_{t}, R_{t^{\prime}}\right\rangle_{R}$, where $R_{t}\left(t^{\prime}\right):=R\left(t, t^{\prime}\right)$, there is an isometric isomorphism between the subspace $V$ and $H_{R}$ generated by the correspondence $\eta_{t} \in V \sim R_{t} \in H_{R}$. Under this isomorphism, $f \sim g \Longleftrightarrow\left\langle\eta_{t}, f\right\rangle_{Q}=\left\langle R_{t}, g\right\rangle$, i.e., $g(t)=(A f)(t) ; P_{V} Q_{s} \sim \eta_{s}^{*}:=A Q_{s}$, where $P_{V}$ is the orthogonal projector from $H_{Q}$ onto $V$.

For $g \in H_{R}$ let $\hat{f}$ be the element in $H_{Q}$ of minimal $H_{Q}$-norm which satisfies the equation $A f=g$. Then $\hat{f} \in V$ and $g \sim \hat{f}$. We have the following representations for $\hat{f}$.

THEOREM 2. If (3) holds and $g \in H_{R}$, then $\hat{f}(s)=\left\langle Q_{s}, \hat{f}\right\rangle_{Q}=$ $\left\langle\eta_{s}^{*}, g\right\rangle_{R}$. Furthermore, if $D\left(A^{*}\right)$ is dense in $Y$, where $A^{*}$ is the adjoint of $A$ considered as an operator from $X$ into $Y$, and if $H_{Q}, H_{R}=A\left(H_{Q}\right)$ possess continuous kernels, then $A_{(Q, R)}^{\dagger} g=Q A^{*}\left(A Q A^{*}\right)_{Y, Y}^{\dagger} g$.

4. Regularization in RKHS. Let $H_{Q}$ and $H_{P}$ be RKHS with norms $\|\cdot\|_{Q}$ and $\|\cdot\|_{P}$ respectively. By a regularized pseudosolution (in RKHS) of the equation $A f=g$, we mean a solution to the variational problem: Find $f_{\lambda} \in H_{Q}$ to minimize

$$
\phi_{g}(f)=\|A f-g\|_{P}^{2}+\lambda\|f\|_{Q}^{2}, \quad \lambda>0
$$

$\left(\phi_{g}(f)\right.$ will be assigned $+\infty$ if $\left.A f-g \notin H_{P}\right)$. In this section, $A$ is a linear operator densely defined on $L_{2}[S]$ into $L_{2}[T]$, and $H_{Q}$ must be chosen so that $A\left[H_{Q}\right]=H_{R}$, where $H_{R}$ is some RKHS contained (as a set) in $L_{2}[T]$, and $H_{P}$ is a subset of $L_{2}[T]$. Assume $g=g_{0}+\xi$ for some $g_{0} \in A\left[H_{Q}\right]$ and some $\xi \in H_{P}$. For $\lambda>0$, let $H_{\lambda P}$ be the RKHS with kernel $\lambda P\left(t, t^{\prime}\right)$, where $P\left(t, t^{\prime}\right)$ is the (continuous) kernel associated with $H_{P}$. We have $H_{P}=H_{\lambda P}$ and $\|\cdot\|_{P}^{2}=\lambda\|\cdot\|_{\lambda P}^{2}$. Let $R(\lambda)=R+\lambda P$, and let $H_{R(\lambda)}$ be the RKHS with kernel $R\left(\lambda ; t, t^{\prime}\right)$. Then (see Aronszajn [1, p. 352]) $H_{R(\lambda)}$ is the Hilbert space of functions of the form $g=g_{0}+\xi$, 
where $g_{0} \in H_{R}$ and $\xi \in H_{P}$. Following Aronszajn [1], we note that this decomposition is not unique unless $H_{P} \cap H_{R}=\{0\}$. The norm on $H_{R(\lambda)}$ is given by

$$
\|g\|_{R(\lambda)}^{2}=\min \left\{\left\|g_{0}\right\|_{R}^{2}+\|\xi\|_{\lambda P}^{2}: g_{0} \in H_{R}, \xi \in H_{P}, g_{0}+\xi=g\right\} .
$$

Theorem 3. Suppose $D\left(A^{*}\right)$ is dense in $Y, H_{Q} \subset D(A)$ and $A$ and $H_{Q}$ satisfy (3). Suppose $H_{Q}, H_{R}$ and $H_{P} \subset Y$ all have continuous kernels. Then for $g \in H_{R(\lambda)}$, the unique minimizing element $f_{\lambda} \in H_{Q}$ of the functional $\phi_{g}(f)$ is given by

$$
f_{\lambda}(s)=\left\langle A Q_{s}, g\right\rangle_{R(\lambda)}=\left(Q A^{*}\left(A Q A^{*}+\lambda P\right)_{Y, Y}^{\dagger} g\right)(s) .
$$

We call the (linear) mapping which assigns to each $g \in H_{R(\lambda)}$ the unique minimizing element $f_{\lambda}$, the regularization operator of the equation $A f=g$.

THEOREM 4. If $H_{P} \cap H_{R}=\{0\}$, then the minimizing element $f_{\lambda}$ of (4) is the solution to the problem: Find $f \in \Omega$ to minimize $\|f\|_{Q}$, where

$$
\Omega=\left\{f \in H_{Q}:\|A f-g\|_{R(\lambda)}=\inf _{h \in H_{Q}}\|A h-g\|_{R(\lambda)}\right\} .
$$

In the setting of this section we have $A\left[H_{Q}\right]=H_{R} \subset H_{R(\lambda)} \subset Y$. Replacing $H_{\widetilde{R}}$ by $H_{R(\lambda)}$ in (2), we obtain

$$
A_{(Q, R(\lambda))}^{\dagger} y=Q^{1 / 2}\left[(R+\lambda P)^{-1 / 2} A Q^{1 / 2}\right]_{(X, Y)}^{\dagger}(R+\lambda P)^{-1 / 2} y
$$

for $y \in O\left(A_{(Q, R(\lambda))}^{\dagger}\right)$. It is helpful to remember that the topology on $H_{R}$ is not, in general, the restriction of the topology of $H_{R+\lambda P}$, with the notable exception of the case $H_{R} \cap H_{P}=\{0\}$. The authors provide elsewhere concrete examples arising in approximation of boundary-value problems where $H_{R}$ is not a closed subspace of $H_{R+\lambda P}$.

We emphasize that if $H_{R} \cap H_{P}=\{0\}$, then $H_{R}$ is a closed subspace of $H_{R+\lambda P}$; in this case the regularization operator is a generalized inverse in an appropriate RKHS (Theorem 4).

\section{Convergence rates of approximate regularized solutions to linear} operator equations. The regularization method of $\S 4$ requires in practice some approximate procedure for solving (4) numerically. The principal result of [7] is the establishment of uniform pointwise convergence of approximate 
solutions obtained by moment discretization of (4). Let $T_{n}=\left\{t_{1}, t_{2}, \cdots\right.$, $\left.t_{n}\right\}$, where $t_{i} \in T, t_{1}<t_{2}<\cdots<t_{n}$. For a generic function $h$ on $T$, let $h_{n}=\left(h\left(t_{1}\right), \cdots, h\left(t_{n}\right)\right)$. Let $P_{n}$ denote the $n \times n$ matrix whose $i j$ th element is $P\left(t_{i}, t_{j}\right)$, and define $\left\|h_{n}\right\|_{P_{n}}=\min \left\{\|e\|: e \in R^{n}, P_{n}^{1 / 2} e=h_{n}\right\}$, if $h_{n} \in R\left(P_{n}\right) ; \infty$ otherwise.

THEOREM 5. Let the operator $A$ be as in $\S 4$. Let $f_{\lambda, n}$ be the minimizing element in $H_{Q}$ of the functional $J_{n}=\left\|(A f)_{n}-g_{n}\right\|_{P_{n}}^{2}+\lambda\|f\|_{Q}^{2}$ for $\lambda>0$. Let $P_{T_{n}}(\lambda)$ be the orthogonal projector of $H_{R(\lambda)}$ onto the. subspace spanned by $\left\{R_{t}(\lambda): t \in T_{n}\right\}$. Then

$$
\begin{aligned}
\left|f_{\lambda}(s)-f_{\lambda, n}(s)\right| & =\left|\left\langle P_{T_{n}}(\lambda) \eta_{s}^{*}, P_{T_{n}}(\lambda) g\right\rangle_{R(\lambda)}\right| \\
& \leqslant\left\|\eta_{s}^{*}-P_{T_{n}}(\lambda) \eta_{s}^{*}\right\|_{R(\lambda)}\left\|g-P_{T_{n}}(\lambda) g\right\|_{R(\lambda)} .
\end{aligned}
$$

Furthermore, let $\Delta=\max \left|t_{i+1}-t_{i}\right|,\left|f_{\lambda}(s)-f_{\lambda, n}(s)\right|=O\left(\Delta^{m}\right)$ or $O\left(\Delta^{2 m}\right)$, depending on smoothness properties of the kernel $R\left(\lambda, t, t^{\prime}\right)$ and the functions $g$ and $\eta_{s}^{*}$. In the particular case when $H_{P} \cap H_{R}=\{0\},\left\{f_{\lambda, n}\right\}$ converges to $A^{\dagger} g$. (An explicit formula for $f_{\lambda, n}$ and furthe: properties of $f_{\lambda}$ are given in [7].)

The results of this paper provide an approach to simultaneous regularization and approximation of (ill-posed) linear operator equations which applies to a large class of operator equations that include boundary-value problems, Fredholm integral equations of the first kind, and integrodifferential equations; see also [2], [10]. The Sobolev spaces $W_{2}^{m}, m \geqslant 0$ are included in the class of spaces considered.

The proofs of the preceding theorems as well as related results and examples will appear in [6] and [7].

\section{REFERENCES}

1. N. Aronszajn, Theory of reproducing kernels, Trans. Amer. Math. Soc. 68 (1950), 337-404. MR 14, 479.

2. M. Golomb and H. F. Weinberger, Optimal approximation and error bounds, On Numerical Approximation (R. E. Langer, editor), Univ. of Wisconsin Press, Madison, Wis., 1959, pp. 117-190.

3. M. M. Lavrent ev, Some improperly posed problems of mathematical physics, Izdat. Sibirsk. Otdel. Akad. Nauk SSSR. Novosibirsk, 1962; English transl., Springer Tracts in Natural Philosophy, vol. 11, Springer-Verlag, Berlin, 1967. MR 36 \#5487; \#7363. 
4. M. Z. Nashed, Generalized inverses, normal solvability, and iteration for singular operator equations, Nonlinear Functional Analysis and Applications (Proc. Advanced Sem. Math. Res. Center, Univ. of Wisconsin, Madison, Wis., 1970), Academic Press, New York, 1971, pp. 311-359. MR 43 \#1003.

5. - Some aspects of regularization and approximation of solutions of illposed operator equations, Proc. Army Numerical Analysis Conference, 1972, pp. 163-181.

6. M. Z. Nashed and Grace Wahba, Generalized inverses in reproducing kernel spaces: An approach to regularization of linear operator equations, SIAM J. Math. Anal. (to appear).

7. - Approximate regularized pseudosolutions of linear operator equations when the data-vector is not in the range of the operator. (to appear).

8. - Rates of convergence and approximate least squares solutions of linear integral and operator equations, Math. Comp. 28 (1974), 69-80.

9. A. N. Tihonov, On methods of solving incorrect problems, Proc. Internat. Congress Math. (Moscow, 1966), “Mir”, Moscow, 1968, pp. 720-722; English transl., Amer. Math. Soc. Transl. (2) 70 (1968), 222-224. MR 38 \#6208.

10. Grace Wahba, $A$ class of approximate solutions to linear operator equations, J. Approximation Theory 9 (1973), 61-77.

SCHOOL OF MATHEMATICS, GEORGIA INSTITUTE OF TECHNOLOGY, ATLANTA, GEORGIA 30332

DEPARTMENT OF STATISTICS, UNIVERSITY OF WISCONSIN, MADISON, WISCONSIN 53706 